\title{
The association of adjusted plasma valproic acid concentration with CYP2C9 gene polymorphism in patients with epilepsy: a systematic review and meta-analysis
}

\author{
Huihui Fang ${ }^{1,2}$, Xiaojuan Wang ${ }^{2}$, Kelu Hou ${ }^{1}$, Ying Zhang ${ }^{1}$, Shuai Shao ${ }^{1}$, Guijie Zhang ${ }^{1}$, Yufei Feng ${ }^{1}$, \\ Lin Huang ${ }^{1 \wedge}$
}

${ }^{1}$ Department of Pharmacy, Peking University People's Hospital, Beijing, China; ${ }^{2}$ Department of Pharmacy, Huainan First People's Hospital, The First Affiliated Hospital of Anhui University of Science and Technology, Huainan, China

Contributions: (I) Conception and design: L Huang; (II) Administrative support: L Huang, Y Feng; (III) Provision of study materials or patients: K Hou, Y Zhang; (IV) Collection and assembly of data: H Fang, X Wang; (V) Data analysis and interpretation: S Shao, G Zhang; (VI) Manuscript writing: All authors; (VII) Final approval of manuscript: All authors.

Correspondence to: Yufei Feng; Lin Huang. Department of Pharmacy, Peking University People's Hospital, Beijing, China.

Email: fenyufei@126.com; huanglin@pkuph.edu.cn.

Background: Valproic acid (VPA) is a common antiepileptic drug used to treat both generalized and partial epilepsy. Although there is increasing evidence to suggest that CYP2C9 gene polymorphisms are associated with interindividual variability of VPA metabolism, the results are debatable. Therefore, in the present study, we conducted a meta-analysis to evaluate the correlation between CYP2C9 gene polymorphisms and adjusted plasma VPA concentration.

Methods: The EMBASE, MEDLINE, and Cochrane Library databases were searched to obtain relevant studies. Eligible articles were reviewed, and data extraction was performed. We calculated $95 \%$ confidence intervals (CIs) and mean differences (MDs) to assess the strength of the relationship of CYP2C9 gene polymorphisms with adjusted plasma VPA concentration.

Results: The meta-analysis included 6 studies involving 847 patients with epilepsy. The pooled analysis showed that the CYP2C9 A1075C (AA vs. AC) polymorphism was related to the adjusted plasma concentration of VPA $\left(\mathrm{P}=0.02, \mathrm{I}^{2}=82 \%\right)$. Additionally, the AC phenotype statistically significantly increased the adjusted plasma VPA concentration in children compared with the mixed age subgroup $(\mathrm{P}=0.04$, $\left.\mathrm{I}^{2}=48 \%\right)$. A similar association was observed between the AC phenotype for Asians $\left(\mathrm{P}<0.00001, \mathrm{I}^{2}=0 \%\right)$ but not for Caucasians ( $\left.\mathrm{P}=0.34, \mathrm{I}^{2}=87 \%\right)$.

Discussion: Age might be a crucial covariate influencing the dosage-adjusted VPA concentration in patients with epilepsy. A reduced VPA dosage may be recommendable for children, particularly Asian children, who are CYP2C9 A1075C AC carriers. Further studies could provide high-quality evidence to confirm the correlation between VPA pharmacokinetics and CYP2C9 A1075C polymorphisms.

Keywords: Valproic acid (VPA); CYP2C9; genetic polymorphism; pharmacokinetics; meta-analysis

Submitted Feb 22, 2021. Accepted for publication Apr 29, 2021.

doi: $10.21037 / \mathrm{atm}-21-1459$

View this article at: http://dx.doi.org/10.21037/atm-21-1459

\footnotetext{
$\wedge$ ORCID: 0000-0003-3264-1374.
} 


\section{Introduction}

Valproic acid (VPA) is a first-line antiepileptic therapy that has been widely applied to treat both generalized and partial seizures (1). However, VPA displays significant interindividual differences and has a narrow therapeutic range, which results in varying therapeutic efficacy and adverse reactions. In recent years, the continuous development of pharmacogenetics has revealed genetic polymorphisms to have pivotal importance in the pharmacodynamics and pharmacokinetics of VPA (2-4). Therefore, genetic factors may contribute to individual VPA pharmacotherapy.

The VPA metabolism in humans involves mainly three pathways: glucuronidation, $\beta$-oxidation in mitochondria, and cytochrome P450 (CYP)-mediated oxidation (5-7). In adults, the conversion of 50\% VPA to VPA-glucuronide conjugate is mediated by glucuronosyl transferases (UGTs) (8). The second route, mitochondrial $\beta$-oxidation, is responsible for approximately $30 \%$ of VPA metabolism, and produces unsaturated VPA metabolites. Elimination of VPA through CYP enzyme catalysis accounts for the final 15-20\% (9). Studies and meta-analyses have reported the effect of $U G T 2 B 7$ polymorphisms on pharmacokinetics in patients with epilepsy who were treated with VPA (10), which suggests that genetic polymorphisms may be a crucial factor in interindividual variability in VPA metabolism.

In children, however, studies have demonstrated CYPmediated VPA catalysis to be the principal route of metabolism (11). The main reason for this difference is that UGTs in children are not matured and exhibit low activity compared with those in adults (12). Secondly, both VPA and its metabolites can inhibit mitochondrial $\beta$-oxidation. Additionally, the CYP-dependent VPA metabolism in children surpasses that in adults and decreases gradually during adolescence. In children, a higher proportion of VPA is metabolized by CYP (13-15). CYP2C9 is the major catalyst for desaturation and hydroxylation to 4-ene-VPA, with $C Y P 2 B 6$ and $C Y P 2 A 6$ making minor contributions. Therefore, it is necessary to explore the relationship between CYP2C9 polymorphism and VPA pharmacokinetics in different age groups.

CYP2C9, which is a major cytochrome $\mathrm{P} 450$ isoform, constitutes approximately $20 \%$ of the total microsome P450 content in the human liver (16). In total, 35 alleles of the CYP2C9 gene, which is located on chromosome 10q24.1, have been described. Several studies on patients with epilepsy have investigated the influence that CYP2C9 genetic polymorphisms have on VPA through blood levels and pharmacokinetic considerations. Among the CYP2C9 variants, $\mathrm{A} 1075 \mathrm{C}$ has been reported to be a vital determinant of individual variability in VPA pharmacokinetics $(17,18)$. However, studies to date have produced inconsistent findings. For instance, it has been shown that the plasma VPA concentration is higher in patients with the AC genotype than in patients with the AA genotype, which is indicative of patients with the AA genotype having a high metabolism (19-22). On the other hand, some studies have failed to observe a significant association between the adjusted plasma concentration of VPA and CYP2C9 A1075C $(23,24)$. Therefore, further clarification of the influence of CYP2C9 gene polymorphism on VPA concentration and other pharmacokinetic parameters, especially in patients of different ages, is essential. Here, we conducted a comprehensive review and meta-analysis to evaluate the correlations between CYP2C9 gene polymorphism and VPA metabolism, which we expect will further guide the individualized use of VPA in clinical practice. We present the following article in accordance with the PRISMA reporting checklist (available at http://dx.doi.org/10.21037/ atm-21-1459).

\section{Methods}

\section{Search strategy}

Systematic searches of the Cochrane Library, EMBASE, and MEDLINE databases were carried out to identify studies published up to December 23, 2020 on the potential correlations between the adjusted plasma VPA concentration and CYP2C9 genetic variants. Key search words included "valproic acid" and "CYP2C9," with the following search strategies in PubMed: $((()(()(($ "valproic acid”(MeSH Terms)) OR "valproic acid”) OR "valproic acid glucuronide") OR "valproate") OR "2-propylpentanoic acid”) OR "2-n-propyl-n-valeric acid”) OR "2-propylpentanoic acid") OR "2-propylpentanoic acid") OR "2-propylvaleric acid”) OR "VPA") AND (((CYP2C9 (MeSH Terms)) OR CYP2C9) OR Cytochrome P450 2C9). There were no restrictions on region, language, age or race of the study participants. The reference lists of original articles and literature reviews were also scanned to avoid the omission of any qualified study.

The abstracts and titles of all articles retrieved by the search engines were read separately by two authors (HHF and $\mathrm{XJW}$ ). Two authors performed the data extraction and quality assessment independently. Any discrepancies between two authors were resolved by discussion until 
reaching a consensus. The final results were reviewed by a senior reviewer $(\mathrm{LH})$.

\section{Exclusion and inclusion criteria}

Studies meeting all of the following inclusion criteria were included in the meta-analysis: (I) assessing the correlations between $C Y P 2 C 9$ and plasma VPA concentration in patients with epilepsy; (II) including AA and AC genotype data and comprehensive statistical indicators; and (III) providing information on the adjusted serum VPA concentration. Studies that did not provide results on the adjusted plasma VPA concentration and those with incomplete data were excluded, as were abstracts, reviews, comments, case reports, and cell or animal studies.

\section{Data extraction and quality assessment}

After eliminating duplicates, two investigators independently extracted detailed data from eligible studies. These data included the name of the first author, publication year, nationality, race, number of participants, dosage-adjusted plasma VPA concentration, genotype distribution, and detection methods. Any disagreements were resolved through discussion between the two investigators or consultation with a $3^{\text {rd }}$ investigator.

The Newcastle-Ottawa quality assessment scale (NOS) is a rating system with a score range of 0-9 used for the quality evaluation of non-randomized studies (25). In the present study, high-quality studies were identified as those with an NOS score of 6 or more. The quality assessment and data extraction were performed independently by two reviewers. Whenever necessary, additional information or raw data were requested from the authors by the reviewers.

\section{Level of evidence}

Evidence from observational studies with different outcomes was rated using the Grading of Recommendations Assessment, Development, and Evaluation (GRADE) framework (26). Inconsistency, risk of bias, imprecision, indirectness, and publication bias may result in a lower evidence level. The GRADE working group evidence grades are as follows: high quality: further research is unlikely to change our confidence in the estimate of effect; moderate quality: further research is likely to have a significant impact on our confidence in the estimate of effect and may change the estimate; low quality: further research is likely to have a vital impact on our confidence in the estimate of effect and is likely to change the estimate; and very low quality: we are extremely uncertain about the estimate.

\section{Statistical analysis}

Statistical analyses were performed using Review Manager 5.2 software. The associations of CYP2C9 polymorphism with adjusted plasma concentration of VPA were evaluated by calculating the mean difference (MD) and corresponding 95\% confidence intervals (CIs). Heterogeneity was estimated using the $\mathrm{I}^{2}$ statistic. Initially, the fixed effects model was applied, but in cases of heterogeneity $\mathrm{I}^{2}>50 \%$, the random effects model was utilized. Subgroup analyses were performed to analyze the impact of ethnicity and age differences on heterogeneity. Further, the deviation of each selected study from the Hardy-Weinberg equilibrium (HWE) was assessed; disequilibrium was considered if $\mathrm{P}<0.05$. Studies not in HWE were subjected to sensitivity analysis, in which each study was sequentially excluded from pooled results with high heterogeneity. Also, publication bias was examined by Egger's and Begg's tests, with a P value of $<0.05$ considered statistically significant.

\section{Results}

\section{Study selection and characteristics}

A flow diagram showing the study selection process is illustrated in Figure 1. All studies were published between 2003 and 2020. An initial online search yielded 63 potentially relevant articles, with 20,31, and 12 articles obtained from Embase, MEDLINE, and the Cochrane Library, respectively. Of these articles, 15 duplicates were excluded. The titles and abstracts of the remaining 48 articles on CYP2C9 polymorphisms and the VPA were then screened against the inclusion criteria. Eventually, 6 eligible original research reports (19-24) involving 847 patients formed the basis of the present meta-analysis. Detailed characteristics of the included studies are presented in Table 1. All participants in the studies were patients with epilepsy, and the majority of the studies were conducted in Asia. For all included studies, the genotype distributions were in HWE.

\section{Quality assessment}

NOS was used to determine the quality of the included 


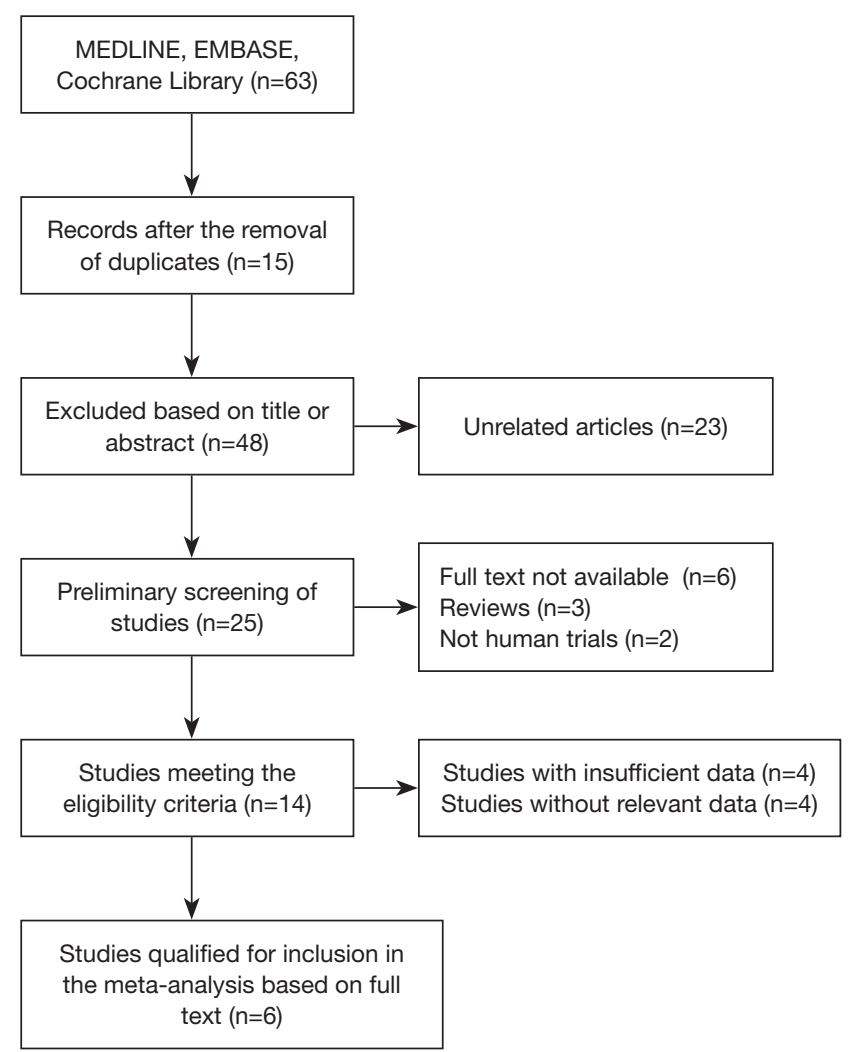

Figure 1 Flow diagram of study selection.

studies. The scores of all 6 studies ranged from 5 to 7 , with only 1 study having a score of less than 6 , indicating a minimal risk of bias and ensuring high quality for the included studies. The quality assessment results are presented in Table 2.

\section{Association between CYP2C9 A1075C polymorphism and adjusted plasma VPA concentration}

\section{Total population}

The results of the meta-analysis are detailed in Figure 2. All 6 studies analyzed the association between CYP2C9 A1075C polymorphism (AA vs. AC) and adjusted plasma VPA concentration (19-24). According to pooled results, AC phenotype carriers exhibited significantly higher adjusted serum concentrations than patients with the AA genotype $[\mathrm{MD}=-0.46,95 \% \mathrm{CI}(-0.84,-0.08), \mathrm{P}=0.02$, $\left.\mathrm{I}^{2}=82 \%\right]$.

\section{Subgroup analysis by age}

Because of the significant heterogeneity observed across the

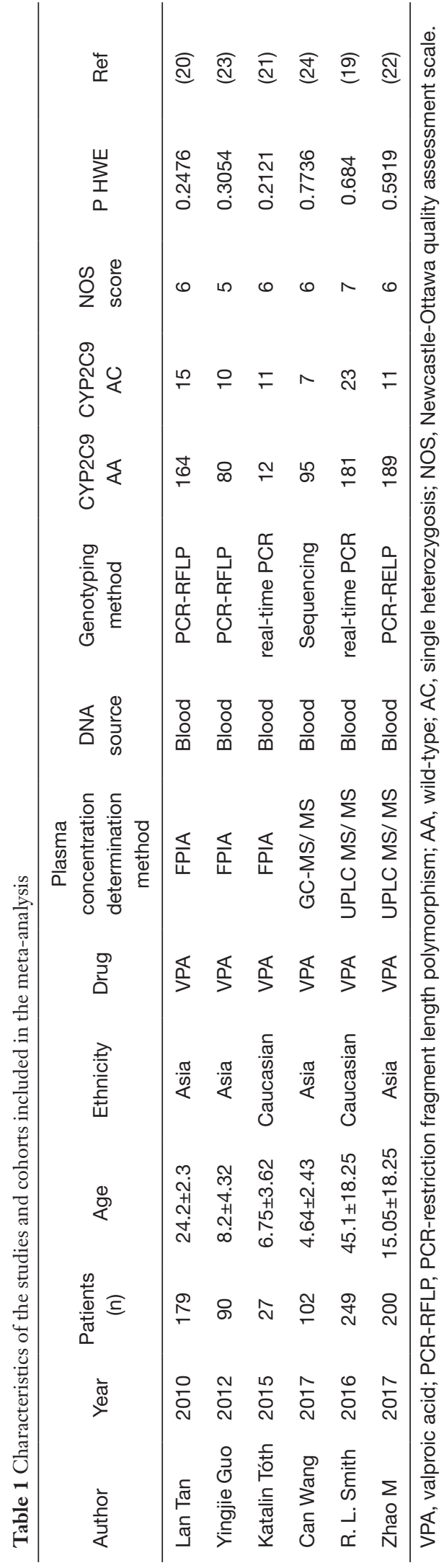


Table 2 Quality assessment of the included studies according to the Newcastle-Ottawa Scale

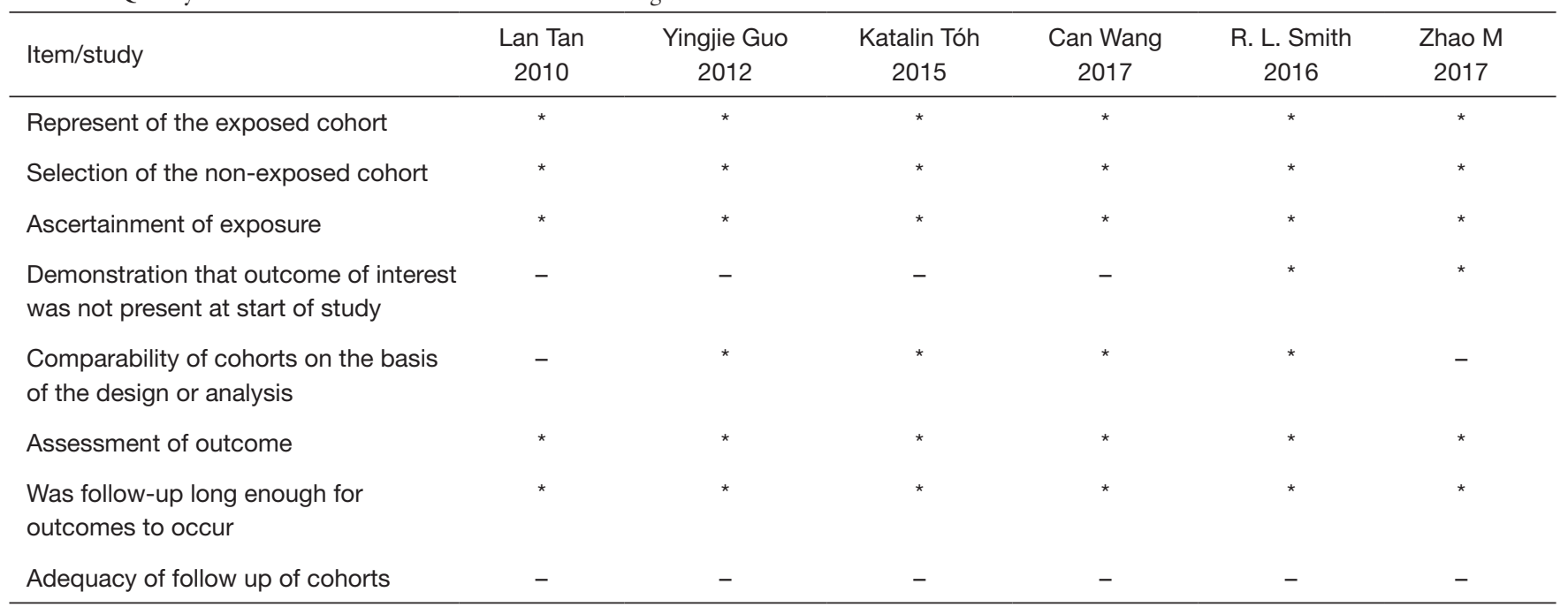

*, a study can be awarded a maximum of one star for each numbered item within the Selection and Exposure categories. A maximum of two stars can be given for Comparability.

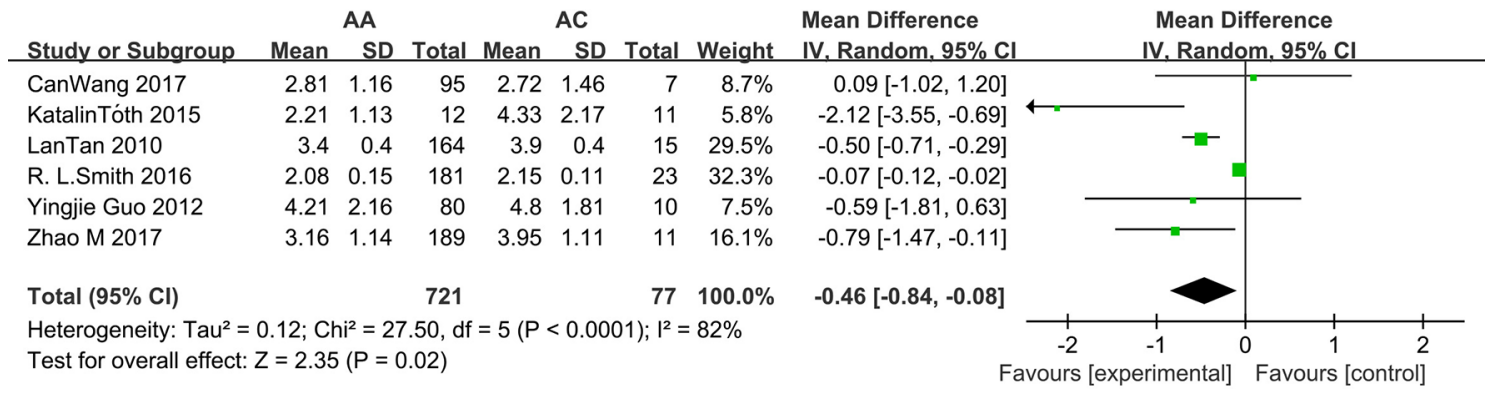

Figure 2 Forest plots for association between CYP2C9 A1075C (AA versus AC) polymorphism and adjusted plasma concentration $(\mu \mathrm{g} / \mathrm{mL}$ per $\mathrm{mg} / \mathrm{kg})$ of VPA. VPA, valproic acid.

studies, age-wise subgroup analyses were further performed. As shown in Figure 3, the AC phenotype exhibited a significantly higher adjusted plasma VPA concentration in children $[\mathrm{MD}=-0.77,95 \%$ CI $(-1.51,-0.03), \mathrm{P}=0.04$, $\left.\mathrm{I}^{2}=48 \%\right]$; however, no effect was observed in the mixed age subgroup $[\mathrm{MD}=-0.27,95 \% \mathrm{CI}(-0.69,0.15), \mathrm{P}=0.20$, $\left.\mathrm{I}^{2}=93 \%\right]$.

\section{Subgroup analysis by race}

Subgroup analysis was performed by race (Figure 4). Among Asians, the adjusted plasma VPA concentration was higher in patients with the AC phenotype than in those with the AA phenotype $[\mathrm{MD}=-0.51,95 \%$ CI $(-0.70,-0.31)$, $\left.\mathrm{P}<0.00001, \mathrm{I}^{2}=0 \%\right]$, whereas stratification of the results in the Caucasian population exhibited no significant effect of the CYP2C9 A1075C polymorphism (AA vs. AC) on adjusted plasma VPA concentrations $(\mathrm{P}=0.34)$. However, as this subgroup analysis included only two studies, its results had a limited influence.

\section{Sensitivity analysis}

To assess the impact of individual data on the pooled MDs, we performed a sensitivity analysis by sequentially omitting each eligible study. The sensitivity analysis results showed that no individual study had a considerable influence on the pooled estimate; thus, the meta-analysis was considered stable. The $\mathrm{P}$ statistic ranged from -0.89 to -0.4 , revealing 


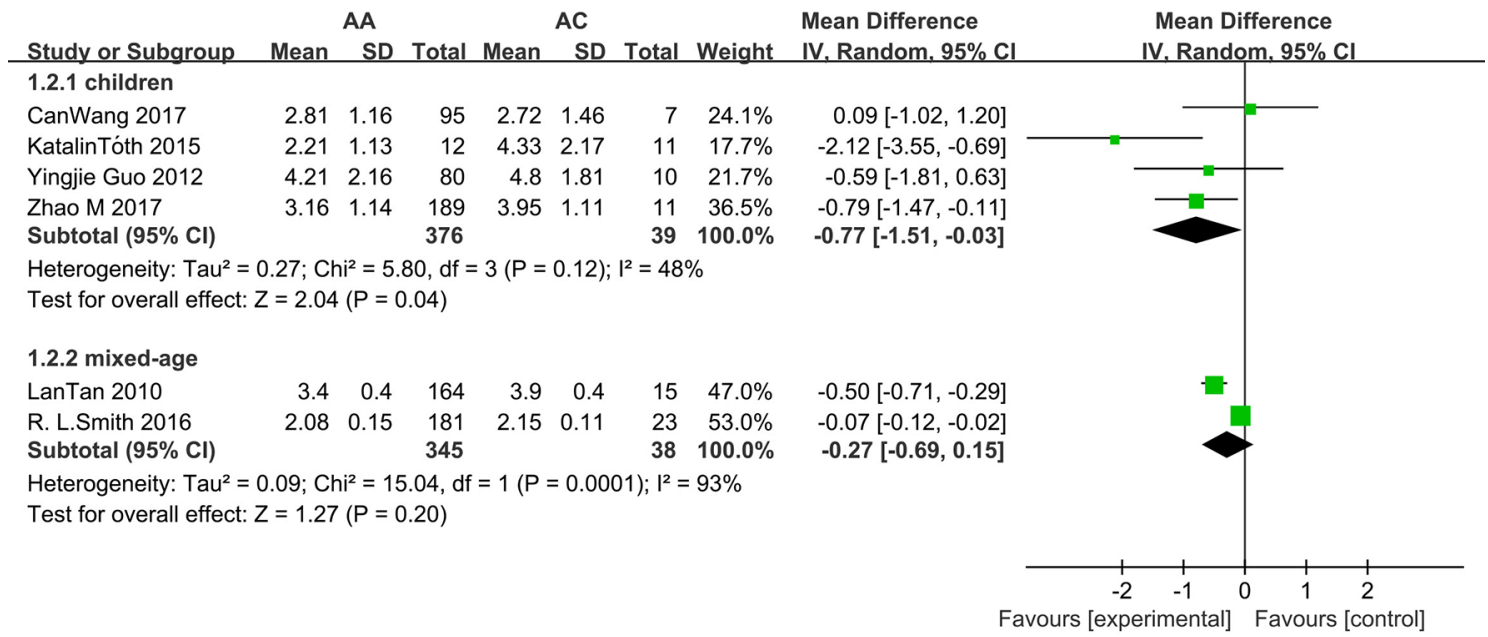

Figure 3 Forest plot of CYP2C9 A1075C polymorphism (AA versus AC) associated with adjusted plasma concentration ( $\mu \mathrm{g} / \mathrm{mL}$ per $\mathrm{mg} / \mathrm{kg}$ ) of VPA according to the age. VPA, valproic acid.

\begin{tabular}{|c|c|c|c|c|c|c|c|c|c|}
\hline Study or Subgroup & \multicolumn{3}{|c|}{ AA } & \multicolumn{3}{|c|}{$A C$} & Weight & $\begin{array}{l}\text { Mean Difference } \\
\text { IV. Random, } 95 \% \mathrm{Cl}\end{array}$ & $\begin{array}{c}\text { Mean Difference } \\
\text { IV. Random, } 95 \% \mathrm{CI}\end{array}$ \\
\hline \multicolumn{10}{|l|}{ 1.3.1 Asian } \\
\hline CanWang 2017 & 2.81 & 1.16 & 95 & 2.72 & 1.46 & 7 & $3.1 \%$ & $0.09[-1.02,1.20]$ & \\
\hline LanTan 2010 & 3.4 & 0.4 & 164 & 3.9 & 0.4 & 15 & $85.9 \%$ & $-0.50[-0.71,-0.29]$ & \\
\hline Yingjie Guo 2012 & 4.21 & 2.16 & 80 & 4.8 & 1.81 & 10 & $2.6 \%$ & $-0.59[-1.81,0.63]$ & \\
\hline $\begin{array}{l}\text { Zhao M } 2017 \\
\text { Subtotal }(95 \% \mathbf{C l})\end{array}$ & 3.16 & 1.14 & $\begin{array}{l}189 \\
528\end{array}$ & 3.95 & 1.11 & $\begin{array}{l}11 \\
43\end{array}$ & $\begin{array}{r}8.4 \% \\
100.0 \%\end{array}$ & $\begin{array}{l}-0.79[-1.47,-0.11] \\
-0.51[-0.70,-0.31]\end{array}$ & \\
\hline \multicolumn{10}{|c|}{$\begin{array}{l}\text { Heterogeneity: } \mathrm{Tau}^{2}=0.00 ; \mathrm{Chi}^{2}=1.81, \mathrm{df}=3(\mathrm{P}=0.61) ; \mathrm{I}^{2}=0 \% \\
\text { Test for overall effect: } \mathrm{Z}=5.08(P<0.00001)\end{array}$} \\
\hline \multicolumn{10}{|l|}{ 1.3.2 Caucasian } \\
\hline KatalinTóth 2015 & 2.21 & 1.13 & 12 & 4.33 & 2.17 & 11 & $43.6 \%$ & $-2.12[-3.55,-0.69]$ & \\
\hline $\begin{array}{l}\text { R. L.Smith } 2016 \\
\text { Subtotal }(95 \% \mathrm{Cl})\end{array}$ & 2.08 & 0.15 & $\begin{array}{l}181 \\
193\end{array}$ & 2.15 & 0.11 & & $\begin{array}{r}56.4 \% \\
100.0 \%\end{array}$ & $\begin{array}{l}-0.07[-0.12,-0.02] \\
-0.96[-2.96,1.03]\end{array}$ & \\
\hline \multicolumn{10}{|c|}{$\begin{array}{l}\text { Heterogeneity: } \mathrm{Tau}^{2}=1.83 ; \mathrm{Chi}^{2}=7.85, \mathrm{df}=1(\mathrm{P}=0.005) ; \mathrm{I}^{2}=87 \% \\
\text { Test for overall effect: } \mathrm{Z}=0.95(\mathrm{P}=0.34)\end{array}$} \\
\hline & & & & & & & & & $\begin{array}{llll}2 & -1 & 0 & 1 \\
\text { erimental] } & \text { Favours }[c\end{array}$ \\
\hline
\end{tabular}

Figure 4 Forest plot of CYP2C9 A1075C polymorphism (AA versus AC) associated with adjusted plasma concentration ( $\mu \mathrm{g} / \mathrm{mL}$ per $\mathrm{mg} / \mathrm{kg}$ ) of VPA according to the ethnicity. The squares and horizontal lines correspond to the study-specific MD and $95 \%$ CI. The area of the squares reflects the weight (inverse of the variance). The diamond represents the MD and 95\% CI. VPA, valproic acid.

no significant values. The results of the sensitivity analysis are shown in Figure 5.

\section{Estimation of publication bias}

Publication bias in the present meta-analysis was examined by Egger's and Begg's tests. No significant publication bias was observed among the included studies through the assessment of the pooled OR (Egger's test: $\mathrm{P}=0.707$; Begg's test: $\mathrm{P}=0.951)$.

\section{Assessment of the level of evidence (GRADE)}

All 6 studies included in the meta-analysis were evaluated using the GRADE framework. The quality of evidence for the overall results was low. For the 4 studies on children (21-24), the evidence presented plausible confounders and was graded as moderate. The 2 studies with participants of 


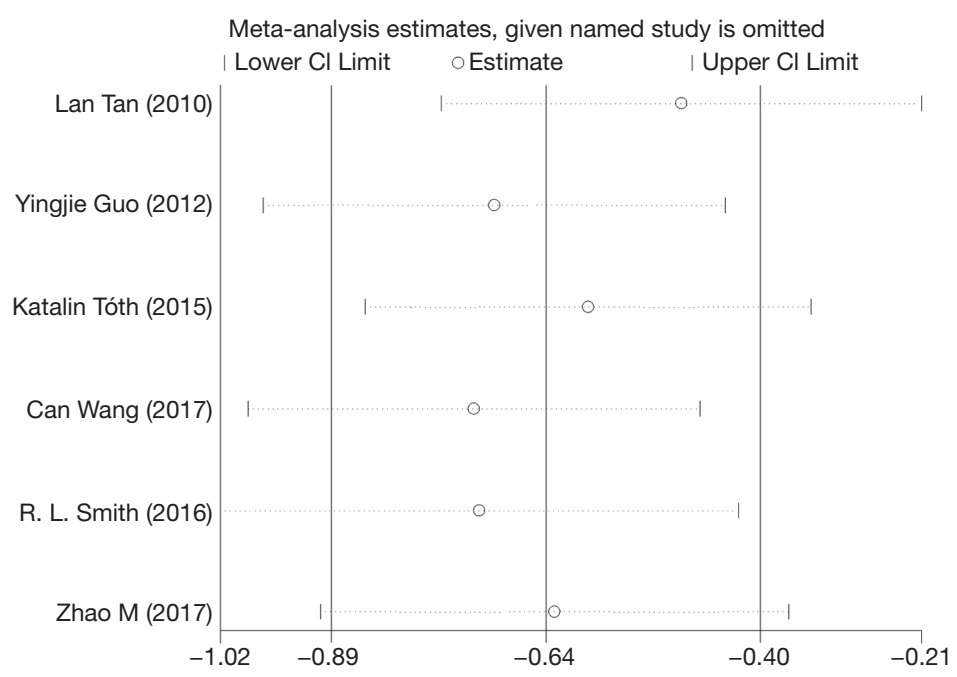

Figure 5 Sensitivity analysis of CYP2C9 A1075C polymorphism associated with adjusted plasma concentration ( $\mu \mathrm{g} / \mathrm{mL}$ per mg/kg) of VPA. VPA, valproic acid.

mixed ages $(19,20)$ were downgraded due to inconsistency $\left(\mathrm{I}^{2}=93 \%\right)$. The evidence level of the 4 studies on Asian patients $(20,22-24)$ was classified as moderate due to a dosage-response gradient increase. The 2 studies involving Caucasian patients $(19,21)$ were downgraded due to inconsistency $\left(\mathrm{I}^{2}=87 \%\right)$ and imprecision. Table 3 presents the GRADE evaluation results.

\section{Discussion}

VPA is a drug which has a narrow therapeutic range and displays significant interindividual variability in plasma concentrations; in turn, this affects its clinical efficacy and safety. Nonlinear pharmacokinetics and the influence of patient characteristics such as sex, age, genetic polymorphisms, weight, and liver disease may account for the variability $(27,28)$. Although the clinical relevance of $U G T$ to VPA metabolism was recently confirmed (10), the correlations between CYP2C9 polymorphisms and VPA metabolism in patients are still poorly understood. Therefore, in the present study, we explored the associations between the CYP2C9 A1075C variants and adjusted plasma VPA concentration levels in patients with different age groups by performing a meta-analysis of existing data. Genetic testing of CYP2C9 might play a role in guiding individualized VPA pharmacotherapy.

Regarding CYP2C9 A1075C allelic variants, the frequency of the CC genotype (loss of function) was $<0.05 \%$ across different racial groups studied. For these patients, Tóth et al. recommended an alternative antiepileptic (nonvalproate) therapy due to the potential for poor valproate metabolism (21), and no individual homozygous mutation for this allele was observed among the other studies.

In the overall analysis of our pooled results, a statistically significant correlation was observed between the variant genotype of CYP2C9 A1075C and adjusted plasma VPA concentration. The present study also exhibited obvious heterogeneity. Therefore, a stratified analysis to detect the source of heterogeneity was conducted, which identified age and race as the sources of the heterogeneity. The subgroup analysis indicated that patients' age might be a pivotal factor influencing the dosage-adjusted VPA concentrations in epilepsy.

A significant association was observed between the CYP2C9 A1075C polymorphism and adjusted plasma VPA concentration in children but not in the mixed age group, which suggested that this polymorphism may be a determinant for adjusted plasma VPA concentration in children. The results also indicated that the adjusted plasma VPA concentration is higher in patients with the AC phenotype than in those with the AA phenotype. Our finding is consistent with the metabolic characteristics of VPA in different age groups. UGTs play a major part in VPA metabolism in adults, but in children, the CYP2C9catalyzed oxidation pathway is the principal metabolic route. Therefore, CYP2C9 genetic polymorphisms could 


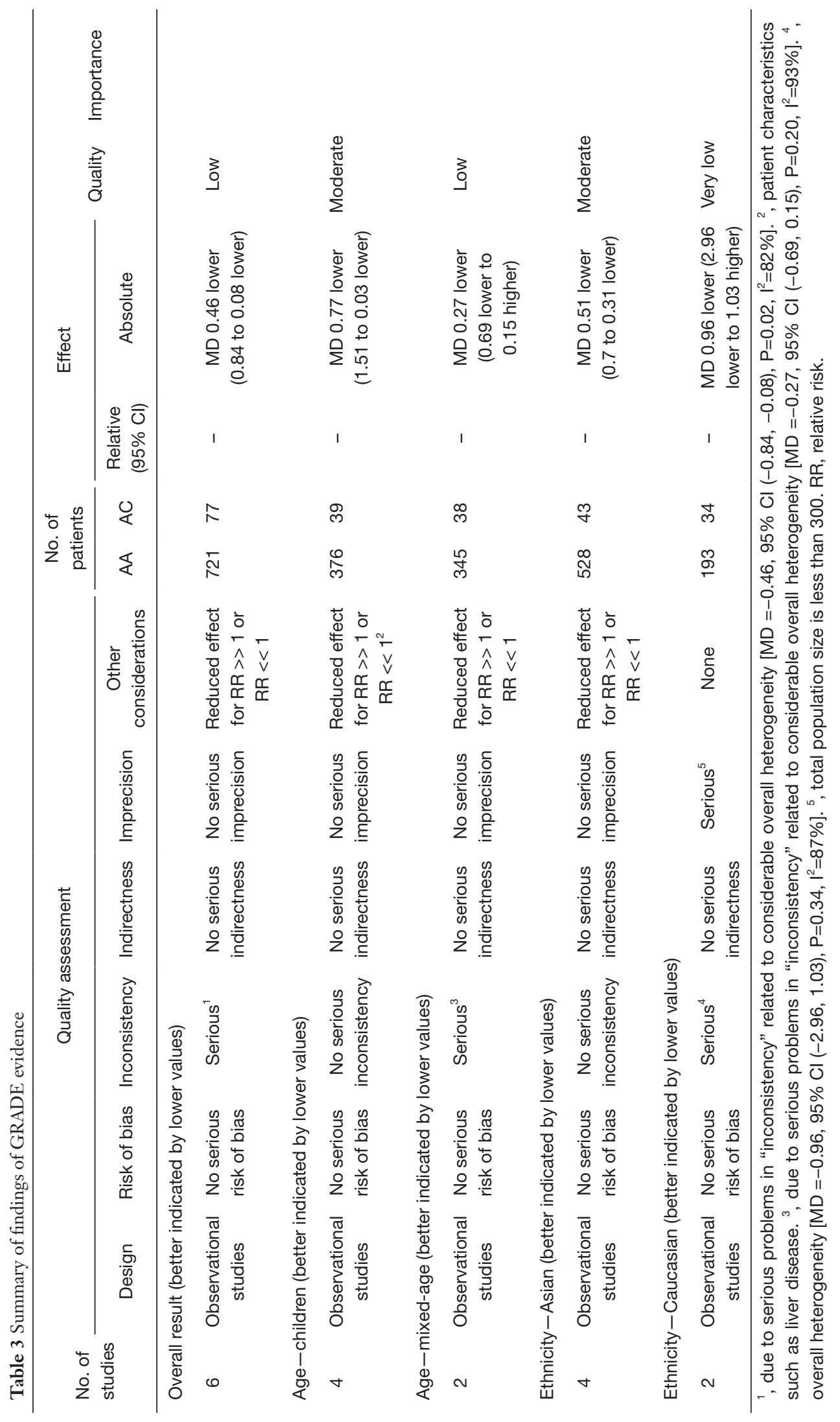


play a prominent role in interindividual variability of VPA metabolism among children. A novel strategy for VPA therapy adjusted according to the CYP2C9 genotype might be developed and recommended for children with epilepsy. Additionally, it is mainly attributed to children under 2 years old, and glucuronidation in children are not matured and exhibit low activity compared with those in adults. The oxidation pathway catalyzed by CYP2C9 will be gradually replaced by UGTs pathway as children grow older. Glucuronidation reaches the normal level after 10-15 years old. Treatment strategies might need to change with the age of children.

Additionally, the subgroup analysis by race revealed that the AC genotype significantly increased the adjusted plasma VPA concentration in Asian patients. The interracial variability in the frequencies of CYP alleles has been well recognized (29). The AC genotype presents with a higher frequency in Asians (11.66\%), than in Caucasians (6.49\%) or Native Americans (2.38\%) (30). Therefore, more attention should be paid to the clinical relevance of the CYP2C9 A1075C alleles in Asian patients.

Despite the substantial analysis and retrieval conducted in the present study, our meta-analysis still has several limitations. First, most of the results demonstrated high heterogeneity, which could be attributable to multiple factors such as differences in therapeutic regimens, age groups, disease staging, sex, race, body mass index, and the methods used for genotype and VPA concentration detection. Subgroup analyses were performed with limited access to the existing data, which may have further influenced the results. Second, the exclusion of several eligible studies due to the absence of available original data might have influenced the results of our meta-analysis. Finally, the small sample sizes of the included studies prevent the generalization of data. Further studies need to be performed to strengthen the current findings.

The present study demonstrated that the age of the patients with epilepsy might be a critical covariate influencing the dosage-adjusted VPA concentration. In the children subgroup, the AC phenotype significantly increased the adjusted plasma concentration of VPA. Similar effects were observed in Asians but not in Caucasians. Therefore, children who carry CYP2C9 A1075C AC, especially Asian children, might require a reduced dosage of VPA. However, there was not enough evidence to determine the specific reduction range for these children. The CYP2C9 A1075C genotype could help clinicians with the adjustment of the VPA dosage prior to treatment initiation, and more attention should be paid to the side effects of VPA in children throughout therapy. Further studies need to be conducted to gather high-quality evidence to confirm the correlation between CYP2C9 A1075C polymorphisms and VPA pharmacokinetics.

\section{Acknowledgments}

Funding: Beijing Municipal Natural Science Foundation (grant No. 7192218) for financial support.

\section{Footnote}

Reporting Checklist: The authors have completed the PRISMA reporting checklist. Available at http://dx.doi. org/10.21037/atm-21-1459

Conflicts of Interest: All authors have completed the ICMJE uniform disclosure form (available at http://dx.doi. org/10.21037/atm-21-1459). The authors have no conflicts of interest to declare.

Ethical Statement: The authors are accountable for all aspects of the work in ensuring that questions related to the accuracy or integrity of any part of the work are appropriately investigated and resolved.

Open Access Statement: This is an Open Access article distributed in accordance with the Creative Commons Attribution-NonCommercial-NoDerivs 4.0 International License (CC BY-NC-ND 4.0), which permits the noncommercial replication and distribution of the article with the strict proviso that no changes or edits are made and the original work is properly cited (including links to both the formal publication through the relevant DOI and the license). See: https://creativecommons.org/licenses/by-nc-nd/4.0/.

\section{References}

1. Ghodke-Puranik Y, Thorn CF, Lamba JK, et al. Valproic acid pathway. Pharmacogenet Genomics 2013;23:236-41.

2. Amini-Shirazi N, Ghahremani MH, Ahmadkhaniha R, et al. Influence of CYP2C9 polymorphism on metabolism of valproate and its hepatotoxin metabolite in Iranian patients. Toxicol Mech Methods 2010;20:452-7.

3. Monostory K, Nagy A, Toth K, et al. Relevance of CYP2C9 Function in Valproate Therapy. Curr Neuropharmacol 2019;17:99-106. 
4. Grover S, Gourie-Devi M, Baghel R, et al. Genetic profile of patients with epilepsy on first-line antiepileptic drugs and potential directions for personalized treatment. Pharmacogenomics 2010;11:927-41. Erratum in: Pharmacogenomics 2010;11:1183-4.

5. Xu S, Chen Y, Zhao M, et al. Population pharmacokinetics of valproic acid in epileptic children: Effects of clinical and genetic factors. Eur J Pharm Sci 2018;122:170-8.

6. Sun YX, Zhuo WY, Lin H, et al. The influence of UGT2B7 genotype on valproic acid pharmacokinetics in Chinese epilepsy patients. Epilepsy Res 2015;114:78-80.

7. Mei S, Feng W, Zhu L, et al. Genetic polymorphisms and valproic acid plasma concentration in children with epilepsy on valproic acid monotherapy. Seizure 2017;51:22-6.

8. Zhang H, Zhang W, Li Y, et al. Correlations between UGT2B7*2 gene polymorphisms and plasma concentrations of carbamazepine and valproic acid in epilepsy patients. Brain Dev 2018;40:100-6.

9. Wang B, Wang J, Huang SQ, et al. Genetic polymorphism of the human cytochrome P450 2C9 gene and its clinical significance. Curr Drug Metab 2009;10:781-834.

10. Wang P, Lin X, Cai W, et al. Effect of UGT2B7 genotypes on plasma concentration of valproic acid: a meta-analysis. Eur J Clin Pharmacol 2018;74:433-42.

11. Büdi T, Toth K, Nagy A, et al. Clinical significance of CYP2C9-status guided valproic acid therapy in children. Epilepsia 2015;56:849-55.

12. Strassburg CP, Strassburg A, Kneip S, et al. Developmental aspects of human hepatic drug glucuronidation in young children and adults. Gut 2002;50:259-65.

13. Siemes H, Nau H, Schultze K, et al. Valproate (VPA) Metabolites in Various Clinical Conditions of Probable VPA-Associated Hepatotoxicity. Epilepsia 1993;34:332-46.

14. Anderson GD. Children versus adults: pharmacokinetic and adverse-effect differences. Epilepsia 2002;43 Suppl 3:53-9.

15. Silva MFB, Aires CCP, Luis PBM, et al. Valproic acid metabolism and its effects on mitochondrial fatty acid oxidation: A review. J Inherit Metab Dis 2008;31:205-16.

16. Van Booven D, Marsh S, McLeod H, et al. Cytochrome P450 2C9-CYP2C9. Pharmacogenet Genomics 2010;20:277-81.

17. Zanger UM, Turpeinen M, Klein K, et al. Functional pharmacogenetics/genomics of human cytochromes P450 involved in drug biotransformation. Anal Bioanal Chem 2008;392:1093-108.
18. Kiang TKL, Ho PC, Anari MR, et al. Contribution of CYP2C9, CYP2A6, and CYP2B6 to Valproic Acid Metabolism in Hepatic Microsomes from Individuals with the CYP2C9*1/*1 Genotype. Toxicol Sci 2006;94:261-71.

19. Smith RL, Haslemo T, Refsum H, et al. Impact of age, gender and CYP2C9/2C19 genotypes on dose-adjusted steady-state serum concentrations of valproic acid a large scale study based on naturalistic therapeutic drug monitoring data. Eur J Clin Pharmacol 2016;72:1099-104.

20. Tan L, Yu J, Sun Y, et al. The influence of cytochrome oxidase CYP2A6, CYP2B6, and CYP2C9 polymorphisms on the plasma concentrations of valproic acid in epileptic patients. Clin Neurol Neurosurg 2010;112: 320-3.

21. Tóth K, Büdi T, Kiss Á, et al. Phenoconversion of CYP2C9 in epilepsy limits the predictive value of CYP2C9 genotype in optimizing valproate therapy. Per Med 2015;12:199-207.

22. Zhao M, Zhang T, Li G, et al. Associations of CYP2C9 and CYP2A6 Polymorphisms with the Concentrations of Valproate and its Hepatotoxin Metabolites and Valproate Induced Hepatotoxicity. Basic Clin Pharmacol Toxicol 2017;121:138-43.

23. Guo Y, Hu C, He X, et al. Effects of UGT1A6, UGT2B7, and CYP2C9 Genotypes on Plasma Concentrations of Valproic Acid in Chinese Children with Epilepsy. Drug Metab Pharmacokinet 2012;27:536-42.

24. Wang C, Wang P, Yang L, et al. Association of CYP2C9, CYP2A6, ACSM2A, and CPT1A gene polymorphisms with adverse effects of valproic acid in Chinese patients with epilepsy. Epilepsy Res 2017;132:64-9.

25. Stang A. Critical evaluation of the NewcastleOttawa scale for the assessment of the quality of nonrandomized studies in meta-analyses. Eur J Epidemiol 2010;25:603-5.

26. Balshem H, Helfand M, Schünemann HJ, et al. GRADE guidelines: 3. Rating the quality of evidence. J Clin Epidemiol 2011;64:401-6.

27. Jiang D, Bai X, Zhang Q, et al. Effects of CYP2C19 and CYP2C9 genotypes on pharmacokinetic variability of valproic acid in Chinese epileptic patients: nonlinear mixed-effect modeling. Eur J Clin Pharmacol 2009;65:1187-93.

28. Price KE, Pearce RE, Garg UC, et al. Effects of Valproic Acid on Organic Acid Metabolism in Children: A Metabolic Profiling Study. Clin Pharmacol Ther 2011;89:867-74.

29. Sychev DA, Shuev GN, Suleymanov SS, et al. 
Comparison of CYP2C9, CYP2C19, CYP2D6, ABCB1, and SLCO1B1 gene-polymorphism frequency in Russian and Nanai populations. Pharmgenomics Pers Med 2017;10:93-9.

30. Céspedes-Garro C, Fricke-Galindo I, Naranjo MEG, et

Cite this article as: Fang H, Wang X, Hou K, Zhang Y, Shao S, Zhang G, Feng Y, Huang L. The association of adjusted plasma valproic acid concentration with CYP2C9 gene polymorphism in patients with epilepsy: a systematic review and meta-analysis. Ann Transl Med 2021;9(10):846. doi: 10.21037/atm-21-1459 al. Worldwide interethnic variability and geographical distribution of CYP2C9 genotypes and phenotypes. Expert Opin Drug Metab Toxicol 2015;11:1893-905.

(English Language Editor: J. Reynolds) 